\title{
Radiation Phenomena in Planetary Entries
}

\author{
Chul Park* \\ Department of Aerospace Engineering, Korea Advanced Institute of Science and Technology, 373-1 Guseong-dong, Yuseong- \\ gu, Daejeon, Korea 305-701; and Division of Aerospace Engineering, Department of Mechanical Engineering, Seoul National \\ University, Gwanhak-no 1, Gwanak-gu, Seoul, 151-741 Korea
}

\begin{abstract}
Radiative heating phenomena occurring in planetary entry flights are reviewed for the purpose of educating those who are not familiar with the problem. How the radiative heat transfer rates to the Apollo entry vehicle were measured and analyzed are first described. Next, the effects of thermo-chemical non-equilibrium on radiation are summarized. Then the radiation problems in entry flights into other planets are reviewed. Finally, unsolved problems are enumerated.
\end{abstract}

Key words: Planetary entries, radiation, nonequilibrium

\section{Introduction}

The problem of radiation phenomenon occurring in high temperature gas was first tackled for the Apollo mission. On return from the moon, the Apollo command module had to reenter Earth's atmosphere at $11 \mathrm{~km} / \mathrm{s}$. At that speed, the flow behind the bow shock wave formed around the command module reached $11,000 \mathrm{~K}$. Radiation emitted at this temperature was greater than the heating rate due to convection. Theoretical calculation and various experiments were conducted to predict the rate of radiative heating of the heatshield.

Since then, all planetary entry missions had to cope with the radiation phenomenon. Because each planet, and sometimes their moon or moons, had different gases in their atmospheres, radiation problem had to be worked out differently for each. In the present review, the past such activities are summarized and the issues central to the phenomena are described

\section{Radiative Heating of Apollo Vehicle}

Theoretical prediction of radiation from high temperature equilibrium air was started in the late 1950s [1-3]. Experimental measurements of radiation were made both in shock tubes [e.g.,
4] and ballistic ranges [e.g., 5]. The heat transfer rates produced by radiation at the time of the reentry flight of the Apollo command module were predicted from the results of such works.

In Fig. 1, The results obtained by Allen [4] in 1965 are compared with the results obtained in 1991 by Sharma et al[6]. As seen here, the early results were overestimating radiative heat flux by about a factor of two. The heat-shield for of the Apollo vehicle was designed with a factor of safety of about two over this overestimated radiative heat transfer rate. As a result, the Apollo's heat-shield was overdesigned probably by a factor of four.

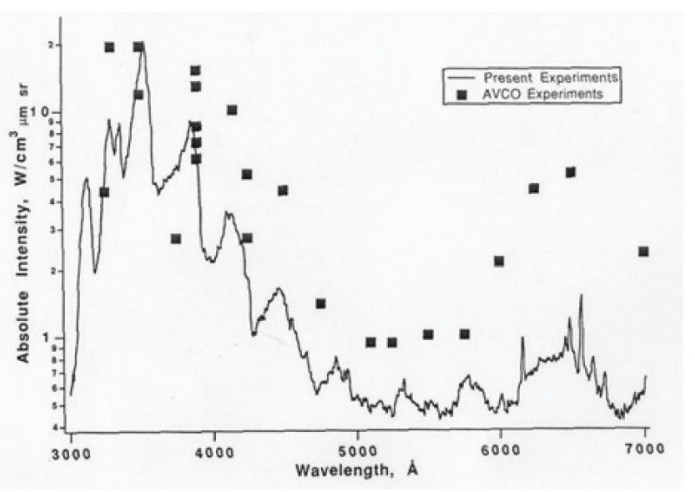

Fig. 1. Spectrum of radiation of air observed in a shock tube at a speed of $10.2 \mathrm{~km} / \mathrm{s}$ and freestream pressure of 0.1 Torr. Square symbols are by Allen [4]. The curves are by Sharma et al [6].
This is an Open Access article distributed under the terms of the Creative Commons Attribution Non-Commercial License (http://creativecommons.org/licenses/by$\mathrm{nc} / 3.0 /$ which permits unrestricted non-commercial use, distribution, and reproduction in any medium, provided the original work is properly cited. (cc * Visiting Professor, Corresponding author : cpark216@kaist.ac.kr 
There was a serious question as to how much of the radiation existing at the edge of the boundary layer, which the shock tube experiment was presumed to be measuring, would reach the heat-shield surface. Apollo's heat-shield was designed to 'perspire.' The heat-shield is made by impregnating a matrix of silica and carbon fibers with an organic resin. The resin vaporized at about $450 \mathrm{deg} \mathrm{K}$ and became a mixture of $\mathrm{H}_{2}$ and carbon particles. The carbon particles were trapped by the matrix, and only $\mathrm{H}_{2}$ molecules left the surface. These $\mathrm{H}_{2}$ molecules mixed with the boundary layer flow formed over the heat-shield surface. The ablation/boundary layer so formed absorbed some of the radiation incident on the layer.

How much absorption occurs in the boundary layer was made known only recently by Park [7]. Fig. 2 shows the spectrum calculated by Park [7]. As seen here, a considerable portion

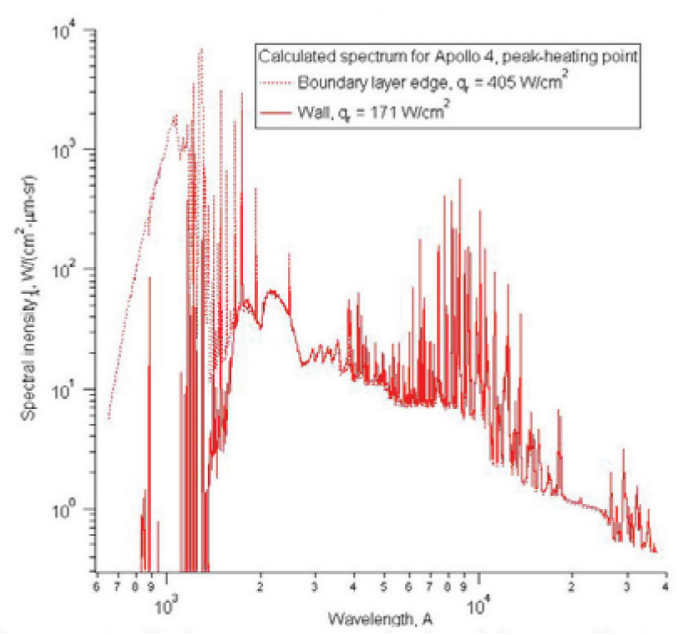

Fig. 2. Radiation spectrum calculated for Apollo 4 at the flight time of $30,032 \mathrm{sec}[7]$

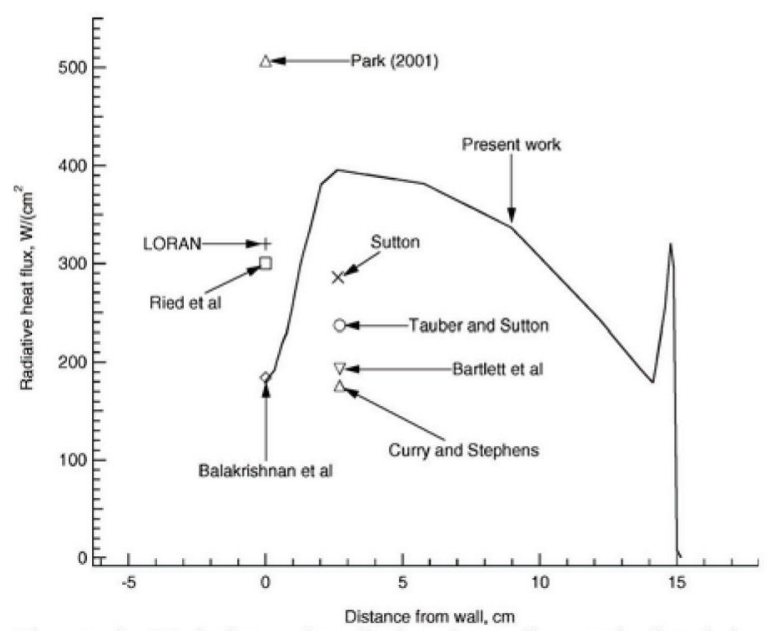

Fig. 3. Variation of radiative heat flux calculated by various investigators compared with the flight data of Ried et al [8]. The present work mentioned in the figure is [7]. of the radiation is absorbed, mostly in the vacuum ultraviolet wavelength range.

The intensity of the radiation falling on the stagnation point of the Apollo entry vehicle was measured in a flight experiment by Ried et al [8]. The radiative heat flux was deduced by Ried et al from the measured intensity values.

Fig. 3 shows Park's calculation [7] with the flight data by Ried et al for the peak-heating point of 30,032 sec. As seen here, at the edge of boundary/ablation layer at around 3 centimeters away from the wall, the radiative heat flux reaches a peak of about $400 \mathrm{~W} / \mathrm{cm}^{2}$. The radiative heat flux so determined are shown as a function of flight time in Fig. 4. At 30,032 sec, the intrinsic convective heat transfer rate was about $120 \mathrm{~W} / \mathrm{cm}^{2}$. By absorbing radiation, convective heat transfer rate increased to $360 \mathrm{~W} / \mathrm{cm}^{2}$. On the other hand, radiative heat transfer rate value of $400 \mathrm{~W} / \mathrm{cm}^{2}$ at the boundary layer edge was reduced to $170 \mathrm{~W} /$ $\mathrm{cm}^{2}$ at the wall because of the absorption [7].

The question as to how much of the radiation incident on a boundary layer edge reaches the wall in general was examined in detail in [9]. It was shown therein that most of the radiative heat flux absorbed in the boundary/ablation layer is converted to convective heat flux. Whatever is not converted into convective heat flux is swept downstream and leaves the flowfield and eventually heats the surrounding atmosphere. The total heat flux received by the heat-shield wall is approximately the sum of the convective heat flux in the absence of radiation and the radiative heat flux at the edge of boundary/ablation layer. The convective heat flux is the well-known Fay-Riddell value corrected for the blowing effect, i.e. reduction of convective heat transfer rate due to mass injection at the wall. Usually, whenever radiation is important, the blowing effect is significant, and so the intrinsic convective heat transfer rate is very small. Therefore, the heat transfer rate felt by the wall is mostly the radiative heat flux at the boundary/ablation layer

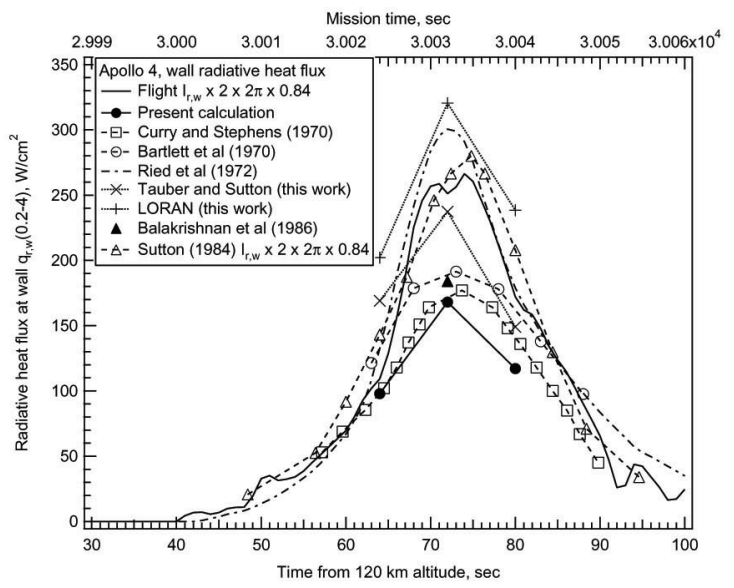

Fig. 4. Variation of the radiative heat flux reaching the wall against flight time [7]. 
edge. This was the case for the Apollo vehicle.

In 1967, Woodward [10] calculated the radiative heat flux emerging from a uniform slab at various flow conditions. Tauber and Sutton [11] used this result to calculate the radiative heat flux at the edge of boundary/ablation layer. In essence, this Tauber-Sutton value of radiative heat flux will be the heat transfer rate felt by the wall in most situations. One qualification: Woodward-Tauber-Sutton values are slightly under-estimating compared with the more recent calculation made in [7]. This is because Woodward's 1967 calculation missed some of the radiation mechanisms because they became known only recently. In any case, this Woodward-Tauber-Sutton procedure is highly recommendable: for any new planetary atmospheres, much time can be saved in predicting the wall heating rate by following this procedure.

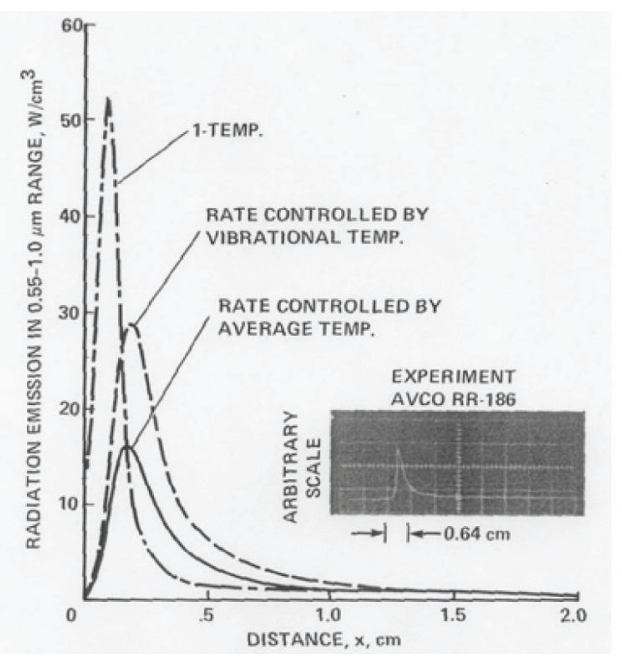

Fig. 5. Nonequilibrium overshoot phenomenon observed in air [4] and its theoretical predictions [9].

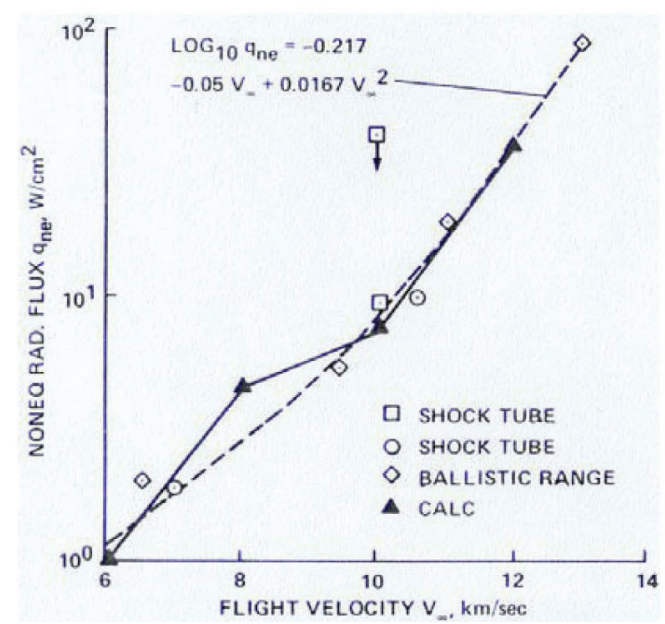

Fig. 6. Nonequilibrium radiative heat flux in air as a function of flight velocity.

\section{Nonequilibrium Radiation}

From these early days, the experimenters noticed that the measured radiation intensities were not those predicted by the theories. Up to about $10 \mathrm{~km} / \mathrm{s}$ of flight speed, there was a region immediately behind the shock wave where radiation was higher than the theoretical calculations based on the assumption of equilibrium. This was called non-equilibrium radiation overshoot phenomenon. The overshoot phenomenon is seen in Fig. 3: immediately behind the shock wave, radiation peaks to a value of about $320 \mathrm{~W} / \mathrm{cm}^{2}$.

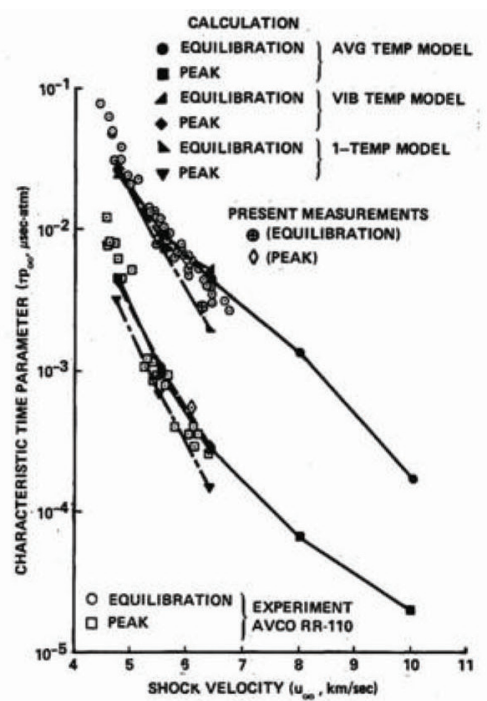

Fig. 7. The time to the peak and time to equilibration of the radiation behind a normal shock wave [4].

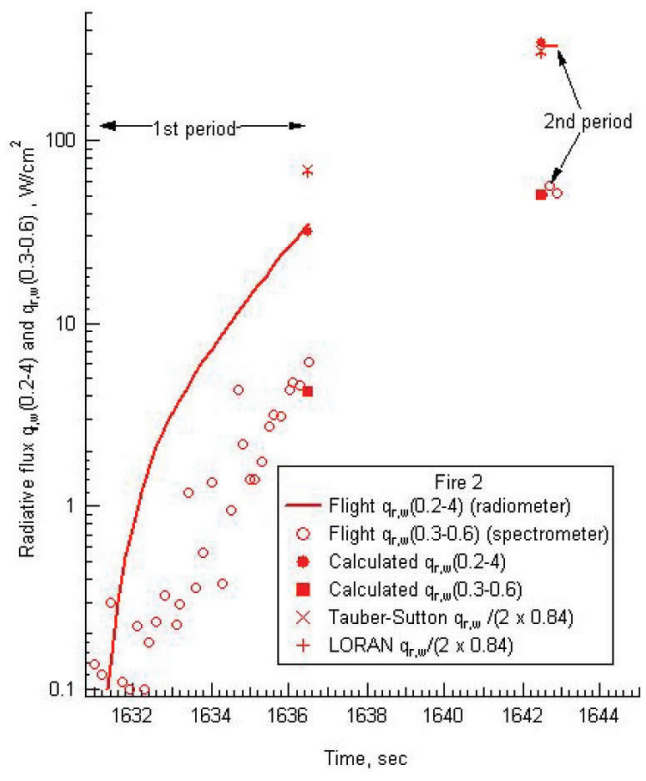

Fig. 8. Radiative heat fluxes for the Fire 2 vehicle [7]. 
In a shock tube experiment, this phenomenon is even more prominent, as shown in the inset in Fig. 5. A new theoretical model called two-temperature model [12] was introduced in order to explain this overshoot phenomenon.

The area under the radiation curve seen in Fig. 5 is known as non-equilibrium radiation intensity. This quantity obeys a binary scaling law so that the area is independent of the freestream density. As a result, the radiative heat flux caused by the non-equilibrium phenomenon is a function only of the flight speed. The calculated values of this quantity are compared with the measurement in Fig. 6. As seen in this figure, prediction is reasonably good. Total radiative heat flux at the boundary/ ablation layer edge is the sum of Woodward-Tauber-Sutton value and the non-equilibrium value.

One question associated with this non-equilibrium radiation phenomenon is about the time taken to reach equilibrium. The measured values are plotted in Fig. 7 and are compared with the calculation made using the two-temperature model [13]. Agreement is good here also.

This non-equilibrium overshoot phenomenon manifests in a flight experiment as well [14-16]. Notable is the flight measurement made in the Project Fire (see Fig. 8). Nonequilibrium radiation overshoot is predicted not only in air but also in Titan's atmosphere [17], Mars' atmosphere [18], and Neptune's atmosphere [19,20]. In Fig. 9, the heat flux values predicted for a Neptune aero-capture flight are shown at the stagnation point [20]. As Fig. 9 shows, radiative heat fluxes expected for Neptune entries are quite high.

The phenomenon does not occur in Venus' atmosphere or Jupiter's atmosphere because the flow tends to be in equilibrium due to high density of their atmospheres.

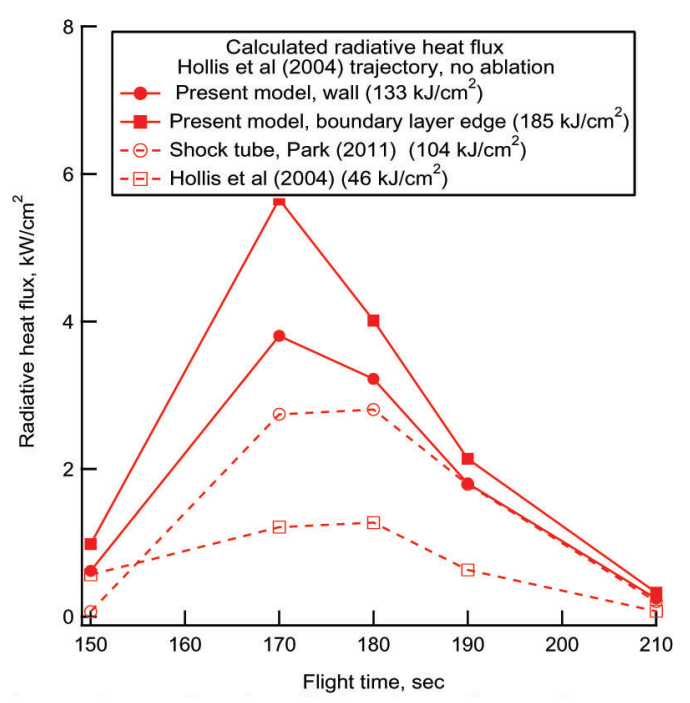

Fig. 9. Predicted radiative heat fluxes for a Neptune aerocapturing vehicle [20].

\section{Radiation in Other Planets}

The atmospheres of the planet Venus and the planet Jupiter are so thick that the flow density behind the bow shock wave becomes high and consequently the flow reaches equilibrium. Radiative heat flux is therefore quite high. In Fig. 10, the radiative heat flux values calculated for the Pioneer-Venus vehicle which entered into Venus are shown [21]. As seen here, the heat flux values are indeed quite high.

The spectrum of radiation in the stagnation region of Galileo Probe [22] at the peak-heating point of $51.16 \mathrm{sec}$ is shown for the stagnation point in Fig. 11. As the figure shows, substantial absorption took place in the boundary/ablation layer. The radiative heating rate was about $30 \mathrm{~kW} / \mathrm{cm} 2$ at the boundary/ ablation layer edge and about $15 \mathrm{~kW} / \mathrm{cm} 2$ at the wall.

The surface recession of the heat-shield for the Galileo Probe vehicle had been overestimated in the stagnation region and underestimated in the frustum region in the original predictions. Matsuyama et al [23] recalculated them accounting for the turbulence generated by ablation [24]. Their results are shown and compared with the flight data in Fig. 12. As seen in the figure, their calculation reproduced the recessions in the frustum region, but overestimated in the stagnation region.

Figure 11. Spectrum of radiation at the stagnation point of Galileo Probe at 51.16 sec point.[22].

More recently, Park [22] recalculated the radiative heat fluxes at the stagnation region of Galileo Probe. He discovered that the equilibrium conditions in the shock layer had not been calculated correctly by the previous investigators. By carrying out the equilibrium calculation correctly, he obtained the stagnation recession values that agreed with the experimental data, as shown in Fig. 12. The temporal changes of surface

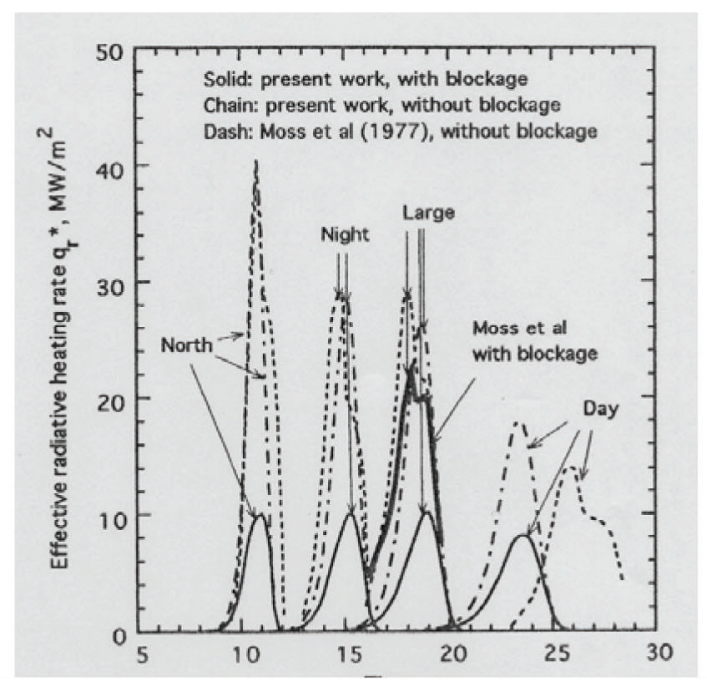

Fig. 10. Radiative heat flux values calculated for the Pioneer-Venus vehicles [21]. 
recession during the entry flight are shown in Fig. 13 and compared with the flight data. As seen in the figure, agreement is quite good.

Radiation phenomenon is important in Neptune aerocapture for another reason: radiation affects chemical reactions [19]. Fig. 14 shows the radiation spectrum for a $30 \mathrm{~km} / \mathrm{s}$ aerocapturing flight. Line radiation is extensive. In particular, Lyman- $\alpha$ line both emits and absorbs. In Fig. 15, the escape factor, the ratio of the radiation power leaving a unit volume to the intrinsic radiation power, for this line is shown. As seen, the escape factor is slightly negative in a region near the shock wave, meaning that absorption is occurring there. The energy absorbed by this line in this region is so large that the rate of ionization of $\mathrm{H}$ is significantly affected. That is, radiationcontrolled chemical reactions occur. In order to determine the non-equilibrium state for a Neptune aero-capturing flight,

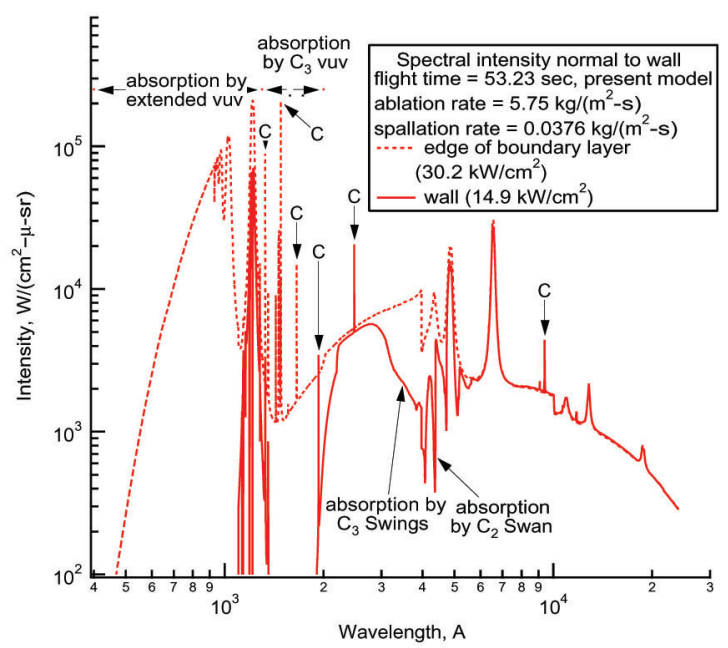

Fig. 11. Spectrum of radiation at the stagnation point of Galileo Probe at $51.16 \mathrm{sec}$ point.[22].

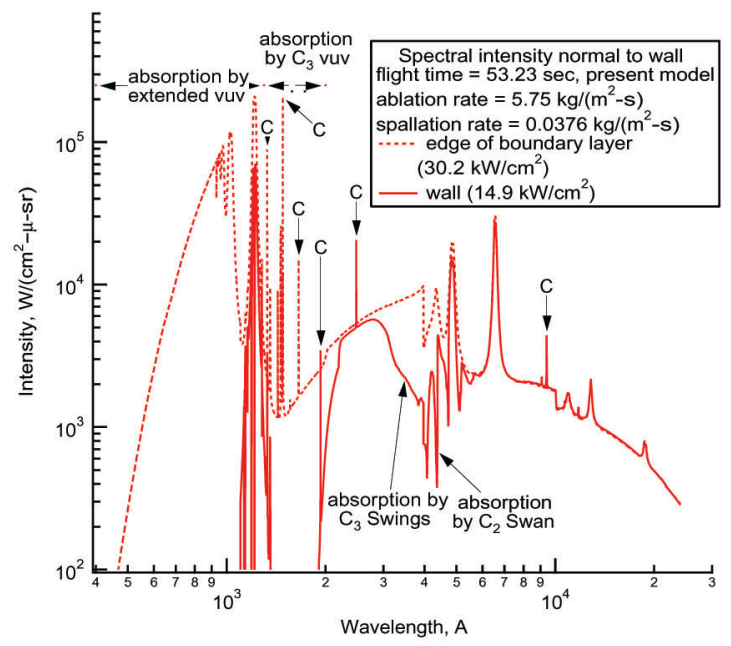

Fig. 12. Surface recession of Galileo Probe. therefore, radiation field must be solved line-by-line and line absorption phenomenon must be accounted for.

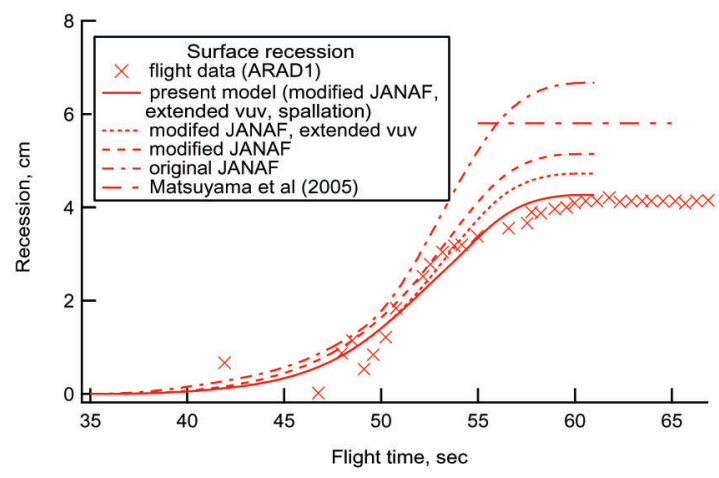

Fig. 13. Temporal variation of surface recession in the stagnation region of Galileo Probe [22].

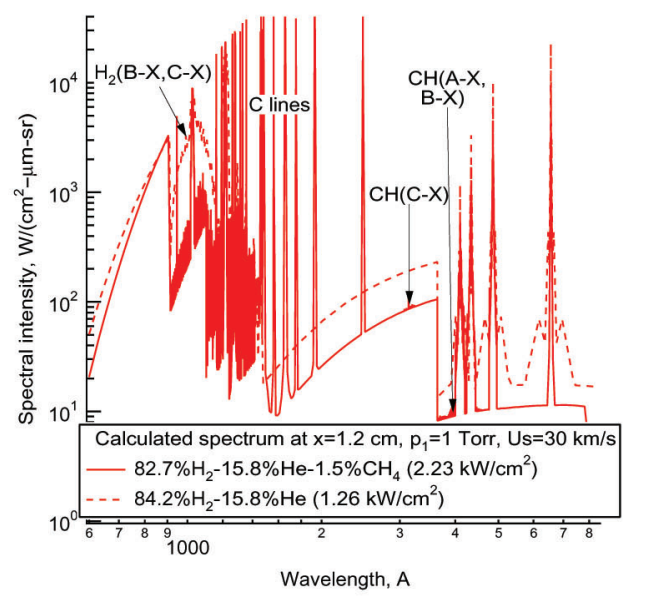

Fig. 14. Spectrum predicted in the shock layer over a Neptune aerocapture vehicle. Freestream pressure $=1$ Torr, flight velocity $=$ $30 \mathrm{~km} / \mathrm{s}$ [19].

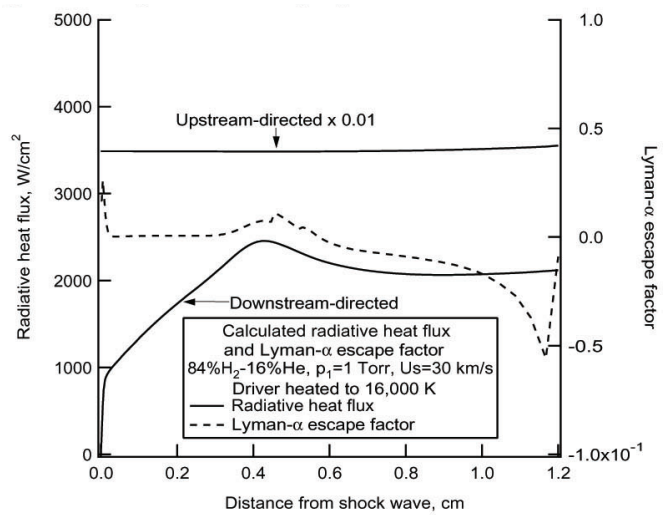

Fig. 15. Radiative heat flux and Lymann- $\alpha$ escape factor in Neptune aerocapture. Freestream pressure $=1$ Torr, flight velocity $=30$ $\mathrm{km} / \mathrm{s}[19]$. 


\section{Unsolved Problems}

The latest of the hyperbolic Earth entry flights were the Stardust [25] and Hayabusa [26]. For both entry flights, spectroscopic measurement was tried. But useful information was gathered only for Stardust. In Fig. 16, the four excitation temperatures measured head-on during the Stardust entry are plotted against altitude. Apparently, $\mathrm{CN}$ and $\mathrm{N}_{2}{ }^{+}$behaved differently. This result has not yet been analyzed.

For the Neptune entry flights mentioned above, the kinetics and radiation mechanisms are yet to be verified experimentally. No experiment has yet been made with a $\mathrm{H}_{2}-\mathrm{He}-\mathrm{CH}_{4}$ mixture. A shock tube capable of delivering $25 \mathrm{~km} / \mathrm{s}$ shock speed with a $\mathrm{H}_{2}-$ $\mathrm{He}-\mathrm{CH}_{4}$ mixture must be constructed to do so without resorting to an electric arc heating of the driver gas. The maximum temperature allowed for the driver is $11,000 \mathrm{~K}$. At higher temperatures, the irradiation from the driver will radiatively heat the driven gas and affect measurements. In an electric-arc driven shock tube, the driver temperature exceeds 11,000 K. As yet, no one is able to build such a shock tube.

One important problem to watch among the unsolved problems of radiative phenomenon in planetary entries is the problem of radiative heating of meteoroids in their entry flights into Earth and other planets. Entry flights of meteoroids are interesting for two reasons: meteoroids inflict damage to the Earth, sometimes devastatingly, and are thought, by some, to have seeded life to the early Earth [27]. There are two types of meteorites: asteroidal and cometary. Asteroidal meteorites enter Earth at a velocity between 12 and $16 \mathrm{~km} / \mathrm{s}$. Cometary meteorites enter at velocities of 30 to $70 \mathrm{~km} / \mathrm{s}$.

At such flight velocities, heat transfer occurs mostly by radiation. For large such meteorites, the shock layer flows around the meteorites will be in equilibrium. Radiative transfer can be calculated using Rosseland approximation. In Fig. 17, Rosseland mean absorption coefficient values for the vapor

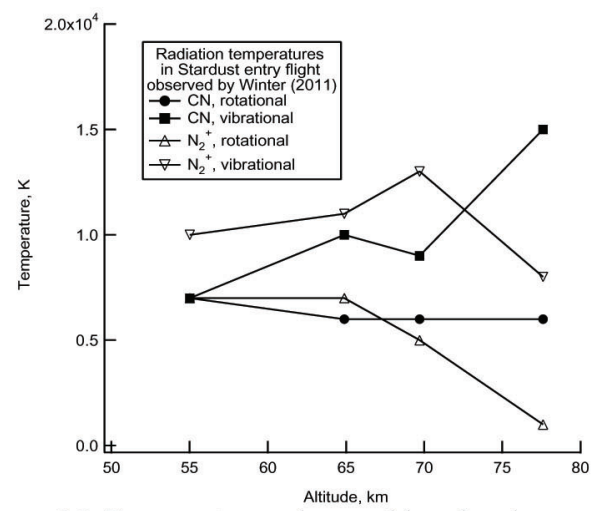

Fig. 16. Temperatures observed in a head-on position for the Stardust entry [25].

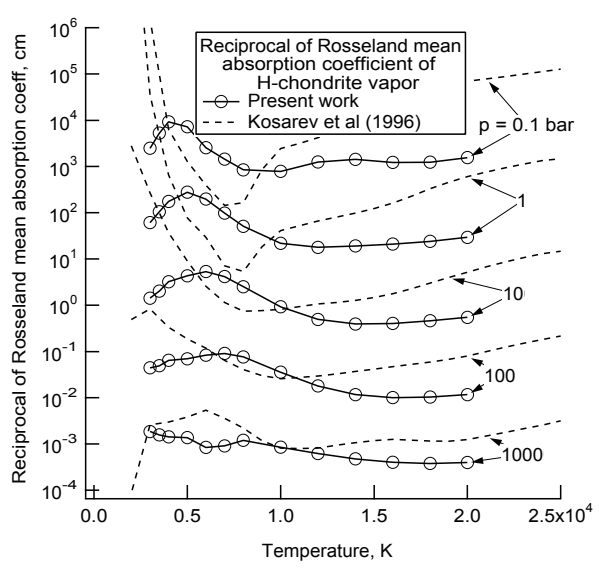

Fig. 17. Rosseland mean absorption coefficient for $\mathrm{H}$-chondrite vapor [29].

of H-chondrites, the most abundant of asteroidal meteorites, calculated recently [28] are shown and compared with those obtained earlier. As seen in the figures, the radiative properties in such a flow regime are not yet fully established.

\section{Concluding Remarks}

The radiation is an important part of the heating process in a planetary entry flight. It involves many physical and chemical disciplines and is quite complicated. There are still unsolved problems in this field. The subject offers a great challenge.

\section{References}

[1] Keck, J. C., Camm, J. C., Kivel, B., and Wentink, T., Jr., "Radiation from Hot Air: Part II", Annals of Physics, Vol. 7, 1959, pp. 1-38.

[2] Armstrong, B. H., Sokoloff, J., Nicholls, R. W., Holland, D. H. Meyerott, R. E., "Radiative Properties of High Temperature Air", Journal of Quantitative Spectroscopy and Radiative Transfer, Vol. 1, No. 1, 1961, pp. 143-169.

[3] Allen, R. A., "Air Radiation Tables: Spectral Distribution Functions for Molecular Band Systems", AVCO-Everett Research Laboratory Research Report 236, 1966.

[4] Allen, R. A., "Nonequilibrium Shock Front Rotat-ional, Vibrational and Electronic Temperature Measure-ments", AVCO-Everett Research Laboratory Research Report 186. 1964.

[5] Page, W. A., and Arnold, J. O., "Shock-Layer Radiation of Blunt Bodies at Reentry Velocities", NASA Technical Report R-193, 1964.

[6] Sharma, S. P., Gillespie, W. D., and Meyer, S. A., "Shock Front Radiation Measurement in Air", AIAA Paper 91-0573, 
1991.

[7] Park, C., "Stagnation-Point Radiation for Apollo 4", Journal of Thermophysics and Heat Transfer, Vol. 18, No. 3, 2004, pp. 348-357.

[8] Ried, R. C., Jr., Rochelle, W. C., and Milhoan, J. D., "Radiative Heating to the Apollo Command Module: Engineering Predictions and Flight Measurement", NASA TM X-58091.

[9] Park, C., "Radiative to Convective Heat Flux Conversion by Boundary Layer Absorption", 47th AIAA Aerospace Science Meeting, Orlando, 2009.

[10] Woodward, H. T., "Predictions of Shock-Layer Radiation From Molecular Band Systems in Proposed Planetary Atmospheres", NASA TN D-3850, 1967.

[11] Tauber, M. E., and Sutton, K., "Stagnation-Point Radiative Heating Relations for Earth and Mars Entries", Journal of Spacecraft and Rockets, Vol. 28, No. 1, 1992, pp. 40-42.

[12] Park, C., "Assessment of Two-Temperature Kinetic Model for Ionizing Nitrogen", Journal of Thermophysics and Heat Transfer, Vol. 2, No. 3, 1988, pp. 8-16.

[13] Park, C., Nonequilibrium Hypersonic Aerothermodynamics, John Wiley and Sons, New York, NY, 1990, pp. 281.

[14] Cauchon, D. L., "Radiative Heating Results From the Fire II Flight Experiment at a Reentry Velocity of 11.4 Kilometers per Second", NASA TM X-1402, 1967.

[15] Arnold, J. O., and Whiting, E. E., "Nonequilibrium Effects on Shock-Layer Radiometry During Earth Entry", Journal of Quantitative Spectroscopy and Radiative Transfer, Vol. 13, No. 9, 1973, pp. 861-870.

[16] Levin, D. A., Candler, G. V., Collins, R. J., Erdman, P. W., Zipf, E. C., and Howlett, L. C., "Examination of Theory for Bow Shock Ultraviolet Rocket Expeirment - 1", Journal of Thermophysics and Heat Transfer, Vol. 8, No. 3, 1994, pp. 447452.

[17] Park, C. S., Bershader, D., and Park, C., "Radiative Emission From the Simulated Shock Layer of the Huygens Probe", Journal of Thermophysics and Heat Transfer, Vol. 10, No. 10, 1996, pp. 563-569.

[18] Lee, E-S., Park, C., and Chang, K-S., "Shock-Tube
Determination of CN Formation Rate in a CO-N2 Mixture", Journal of Thermophysics and Heat Transfer, Vol. 21, No. 1, 2007, pp. 50-56.

[19] Park, C., "Nonequilibrium Chemistry and Radiation for Neptune Entry", Journal of Spacecraft and Rockets, Vol. 48, No. 6, 2011, pp. 897-903.

[20] Park, C., "Viscous Shock Layer Calculation of Stagnation-Region Heating Environment", to be published in Journal of Spacecraft and Rockets.

[21] Park, C., and Ahn, H. K., "Stagnation-Point Heat Transfer Rates for Pioneer-Venus Probes", Journal of Thermophysics and Heat Transfer, Vol. 13, No. 1, 1999, pp. 33-41.

[22] Park, C., "Stagnation-Region Heating Environment of the Galileo Probe", Journal of Thermophysics and Heat Transfer, Vol. 23, No. 3, 2009, pp. 417-424.

[23] Matsuyama, S., Ohnishi, N., Sasoh, A., and Sawada, K., "Numerical Simulation of Galileo Probe Entry Flowfield with Radiation and Ablation", Journal of Thermophysics and Heat Transfer, Vol. 19, No. 1, 2005, pp. 28-35.

[24] Park, C., "Injection-Induced Turbulence in Stagnation-Point Boundary Layers", AIAA Journal, Vol. 22, No. 2, 1984, pp. 219-225.

[25] Winter, M. W., and Trumble, K. A., "Near-Ultraviolet Emission Spectroscopy During an Airborne Observation of the Stardust Reentry", Journal of Spacecraft and Rockets, Vol. 48, No. 1, 2011, pp. 59-71.

[26] Fujita, K., Takayanagi, H., Suzuki, T., Yanagisawa, T., Kurosaki, H., and Yamamoto, M., "Optical Tracking and Spectroscopic Measurement of Hayabusa Capsule Reentry Fireball", 49th AIAA Aerospace Sciences Meeting, Orlando, 2011.

[27] Park, C., "Hypersonic Aerothermodynamics: Past, Present, and Future", International Journal of Aerospace Sciences, Vol. 14, No. 1, 2013, pp. 1-10.

[28] Park, C., "Rosseland mean Opacities of Air and H-Chondrite Vapor in Meteor Entry Problems", to be published in Journal of Quantitative Spectroscopy and Radiative Transfer. 